\title{
Do Antiretroviral Drugs Protect From Multiple Sclerosis by Inhibiting Expression of MS-Associated Retrovirus?
}

\author{
Elena Morandi ${ }^{1,2}$, Radu Tanasescu ${ }^{1,3,4}$, Rachael E. Tarlinton ${ }^{5}$, \\ Dumitru Constantin-Teodosiu ${ }^{6}$ and Bruno Gran ${ }^{1,3 *}$
}

${ }^{1}$ Clinical Neurology Research Group, Division of Clinical Neuroscience, University of Nottingham School of Medicine, Nottingham, United Kingdom, ${ }^{2}$ Centre de Physiopathologie de Toulouse Purpan, UPS, INSERM, CNRS Université de Toulouse, Toulouse, France, ${ }^{3}$ Department of Neurology, Nottingham University Hospitals NHS Trust, Nottingham, United Kingdom, ${ }^{4}$ Division of Clinical Neurosciences, Department of Neurology, Colentina Hospital, University of Medicine and Pharmacy Carol Davila, Bucharest, Romania, ${ }^{5}$ School of Veterinary Medicine and Science, University of Nottingham, Nottingham, United Kingdom, ${ }^{6}$ School of Life Sciences, School of Medicine, University of Nottingham, Nottingham, United Kingdom

\section{OPEN ACCESS}

Edited by:

Robert Weissert,

University of Regensburg, Germany

Reviewed by:

Chiara Cordiglieri,

Istituto Nazionale Genetica Molecolare

(INGM), Italy

Seema Kalra,

University Hospitals of North Midlands NHS Trust, United Kingdom

*Correspondence:

Bruno Gran bruno.gran@nuh.nhs.uk

Specialty section:

This article was submitted to

Multiple Sclerosis and

Neuroimmunology,

a section of the journal

Frontiers in Immunology

Received: 09 July 2018 Accepted: 13 December 2018 Published: 22 January 2019

Citation:

Morandi E, Tanasescu R, Tarlinton RE, Constantin-Teodosiu D and Gran B (2019) Do Antiretroviral Drugs Protect

From Multiple Sclerosis by Inhibiting Expression of MS-Associated

Retrovirus? Front. Immunol. 9:3092. doi: 10.3389/fimmu.2018.03092
The expression of human endogenous retroviruses (HERVs) has been associated with Multiple Sclerosis (MS). The MS-related retrovirus (MSRV/HERV-W) has the potential to activate inflammatory immunity, which could promote both susceptibility and progression toward MS. A connection between HERVs and MS is also supported by the observation that people infected with the human immunodeficiency virus (HIV) may have a lower risk of developing MS than the HIV non-infected, healthy population. This may be due to suppression of HERV expression by antiretroviral therapies (ART) used to treat HIV infection. In this pilot study, we compared RNA expression of the envelope gene of MSRV/HERV-W, as well as Toll-like receptors (TLR) 2 and 4, in a small cohort of $\mathrm{HIV}+$ patients with MS patients and healthy controls $(\mathrm{HC})$. An increased expression of MSRV/HERV-Wenv and TLR2 RNA was detected in blood of MS patients compared with HIV patients and HC, while TLR4 was increased in both MS and HIV patients. There was, however, no difference in MSRV/HERV-Wenv, TLR2 and TLR4 expression between ART-treated and -untreated HIV patients. The viral protein Env was expressed mainly by $B$ cells and monocytes, but not by $T$ cells and EBV infection could induce the expression of MSRV/HERV-Wenv in Lymphoblastoid cell lines (LCLs). LCLs were therefore used as an in vitro system to test the efficacy of ART in inhibiting the expression of MSRV/HERV-Wenv. Efavirenz (a non-nucleoside reverse transcriptase inhibitor) alone or different combined drugs could reduce MSRV/HERV-Wenv expression in vitro. Further, experiments are needed to clarify the potential role of ART in protection from MS.

Keywords: multiple sclerosis, human endogenous retroviruses, MSRV, HIV, antiretroviral treatments

\section{INTRODUCTION}

Multiple Sclerosis (MS) is a demyelinating disease of the central nervous system (CNS). The cause of MS is not known, but genetic linkage studies and the beneficial effect of immunomodulatory treatments indicate that the disease is immune-mediated (1). Activation of autoimmune processes in MS results from the interaction of genetic and environmental factors 
(which include Herpes viral infections, smoking, and vitamin D deficiency) (2). An additional risk factor that has been identified is the expression of human endogenous retroviruses (HERVs) of the HERV-W family $(3,4)$.

HERVs are viruses that have integrated into human germ line cells between 30 and 70 million years ago, becoming part of human DNA and being then transmitted through subsequent generations. Indeed, almost $8 \%$ of the human genome is constituted of groups of HERVs, ranging in copy number from one to many thousands (5).

It has been reported that endogenous retroviruses can engage the innate immune system, and in particular Toll-like receptors (TLRs) (6). Both in vitro and in vivo experiments have illustrated the immunopathogenicity induced by MS-associated retrovirus (MSRV/HERV-W) proteins through direct interaction with TLR4 (7-9). Upon TLR4 engagement by HERVs, signaling pathways are activated that lead to secretion of pro-inflammatory cytokines, such as IL-1 $\beta$, IL-6, and TNF- $\alpha$ (7).

Comorbidity of Human Immunodeficiency Virus (HIV) and MS is very rare (10). Gold et al. examined the association between HIV and MS using an English medical database with a cohort of 21,207 HIV-positive patients and 5,298,496 controls stratified by age, sex, year of first hospital admission, a region of residence, and socioeconomic status. They calculated that the risk rate ratio of developing MS was significantly lower in people infected than in those not infected by HIV ( $0.38 ; 95 \%$ CI 0.15 to 0.79$)$ (10). The authors discussed two different hypotheses that could explain this inverse correlation. The first is related to the HIV viral infection itself. HIV is an infectious retrovirus that if left untreated causes suppression of the immune system, eventually leading to lifethreatening infections and cancers. Primarily, HIV targets the CD4+ lymphocytes cells, which are in their turn thought to be involved in the pathogenesis of MS. The reduction of CD4+ T cells in infected people could, therefore, lower any autoimmune response against the CNS. However, clinical cases where patients have developed MS or CNS demyelinating disorders after HIV infection have been reported (11-13), suggesting thereby that HIV may not protect from MS. Conversely, as HIV-infected MS patients who received Antiretroviral Therapy (ART) had a less severe clinical course of MS $(11,14,15)$, this would suggest that if an inverse association between the infection and MS exists, it may in fact be due to the effect of ART on MS. Unfortunately, Gold et al. did not report which patients were taking retroviral treatments, but rather assumed that most of the patients were on ART, as one would expect to be the case for developed countries (10).

Antiretroviral drugs are classified based on the phase of the retroviral life-cycle that each drug targets. Typically, a combination of drugs from different classes are used to optimize their efficacy in the treatment of HIV infection [termed ART, or combination anti-retroviral therapy (cART)]. These antiretroviral therapies act not only against HIV but probably also inhibit endogenous retroviruses, which thereby could potentially prevent the development of MS. In line with this contention, a phase II clinical trial (INSPIRE) studying the effect of the integrase (enzyme that inserts the viral genome into the DNA of the host cell) inhibitor Raltegravir on relapsing-remitting
(RR)-MS patients has been completed. Unfortunately, this trial did not show any impact of the drug on MS inflammatory activity detected by MRI (16). However, as HERVs are already integrated into the genome, they may not be affected by an integrase inhibitor.

In the current study, we aimed to test the hypothesis that ART can reduce the expression of HERVs. A small cohort of HIV+ patients who were or were not on ART was recruited to study in vivo the effect of antiretroviral drugs on the expression of human MSRV/HERV-W. In parallel, the same classes of the drug were used to test their efficacy in MSRV/HERV-W inhibition in vitro, treating Lymphoblastoid cell lines (LCL).

\section{MATERIALS AND METHODS}

\section{Human Blood Samples}

Blood samples from patients attending Nottingham University Hospitals NHS Trust were collected in PAXgene Blood RNA tubes (Qiagen) and directly stored at $-80^{\circ} \mathrm{C}$ and later used for gene expression analysis. MS diagnosis was established according to McDonald's criteria (17), and HIV infection was certified at the Microbiology Department at the same hospital. All patients and HCs signed informed consent for which ethical approval was obtained. Patients and HC age, gender and clinical status are presented in Table 1.

\section{RNA Extraction and cDNA Synthesis}

Total RNA was purified using the PAXgene Blood RNA kit (Qiagen) following the manufacturer's instructions. The RNA was treated with DNase to remove trace amounts of bound DNA. After the wash steps, RNA was extracted in the elution buffer provided with the kit and stored at $-80^{\circ} \mathrm{C}$. RNA concentration was determined by measuring the absorbance at $260 \mathrm{~nm}$ using NanoDrop ND-100 (Thermo Scientific). For making cDNA, $0.5 \mu \mathrm{g}$ RNA samples, $2 \mu \mathrm{l}$ Random hexamers and $1 \mu \mathrm{l}$ dNTPs mix (stock solution $10 \mathrm{mM}$ ) (all Promega) were mixed, followed by a $5 \mathrm{~min}$ incubation at $65^{\circ} \mathrm{C}$ for first strand cDNA synthesis. A master mix containing RNase inhibitor (Promega, United Kingdom), DTT (0.1 M) and 5X First-Strand Buffer was added, along with Superscript III RT (220 U/ $\mu$ l) (all from Invitrogen, United Kingdom). Negative controls replacing the RNA template or the RT with DNase/RNase free $\mathrm{H}_{2} \mathrm{O}$ were included. Samples were incubated as followed: $5 \mathrm{~min}$ at $95^{\circ} \mathrm{C}$, $60 \mathrm{~min}$ at $50^{\circ} \mathrm{C}$, and $25 \mathrm{~min}$ at $70^{\circ} \mathrm{C}$. cDNA was stored at $-80^{\circ} \mathrm{C}$.

\section{Real-Time RT-PCR}

After titration of different cDNA dilutions, 1:2 DNase/RNase free $\mathrm{H}_{2} \mathrm{O}$ dilution was selected. Relative quantification was performed using Hydroxymethylbilane Synthase (HMBS) as housekeeping gene. Nine hundred nanomolar of probe/forward and reverse primer mix of MSRV//HERV-Wenv (designed as reported previously in the literature (18), TLR4, TLR2, and HMBS (TaqMan, Invitrogen) were used. All samples were run in duplicates. RT- PCR reactions were performed using the 7900HT Fast Real-Time PCR system (Applied Biosystems) in 96-well plates. The following incubation protocol was imposed: $10 \mathrm{~min}$ at $95^{\circ} \mathrm{C}$ and 40 cycles of $10 \mathrm{~s}$ at $95^{\circ} \mathrm{C}$ followed by $30 \mathrm{~s}$ at $60^{\circ} \mathrm{C}$. 
TABLE 1 | Information on subjects in the study.

\begin{tabular}{|c|c|c|c|c|c|c|c|}
\hline Subject & Age & Gender & Ethnicity & & & & \\
\hline HC 1 & 32 & M & Caucasian & & & & \\
\hline $\mathrm{HC} 2$ & 44 & $\mathrm{~F}$ & Caucasian & & & & \\
\hline $\mathrm{HC} 4$ & 38 & $\mathrm{~F}$ & Caucasian & & & & \\
\hline HC 5 & 27 & M & Caucasian & & & & \\
\hline HC 6 & 54 & M & Caucasian & & & & \\
\hline $\mathrm{HC} 9$ & 51 & $\mathrm{~F}$ & Jamaican & & & & \\
\hline HC 10 & 42 & M & Caucasian & & & & \\
\hline HC 11 & 27 & M & Caucasian & & & & \\
\hline $\mathrm{HC} 12$ & 44 & M & & & & & \\
\hline $\mathrm{HC} 13$ & 60 & $\mathrm{~F}$ & Caucasian & & & & \\
\hline HC 14 & 44 & $\mathrm{~F}$ & Caucasian & & & & \\
\hline HC 15 & 55 & F & Caucasian & & & & \\
\hline HC 21 & 27 & M & Caucasian & & & & \\
\hline HC 22 & 44 & $\mathrm{~F}$ & Caucasian & & & & \\
\hline Subject & Age & Gender & Ethnicity & Disease course & Current D & & Disease duration (years) \\
\hline \multicolumn{8}{|c|}{ (B) INFORMATION ON MS PATIENTS } \\
\hline MS 1 & 41 & $\mathrm{~F}$ & Caucasian & $\mathrm{RR}$ & no & & 5 \\
\hline MS 2 & 56 & $\mathrm{~F}$ & Caucasian & & no & & 8 \\
\hline MS 3 & 38 & $\mathrm{~F}$ & Caucasian & $\mathrm{RR}$ & no & & 3 \\
\hline MS 4 & 54 & M & Caucasian & $\mathrm{RR}$ & no & & 6 \\
\hline MS 5 & 55 & $\mathrm{~F}$ & & $\mathrm{SP}$ & no & & \\
\hline MS 6 & 46 & $\mathrm{~F}$ & & $\mathrm{SP}$ & no & & \\
\hline MS 7 & 44 & $\mathrm{~F}$ & & $\mathrm{RR}$ & no & & \\
\hline MS 8 & 46 & $\mathrm{~F}$ & & $\mathrm{RR}$ & no & & \\
\hline MS 18 & 48 & $\mathrm{~F}$ & Caucasian & & no & & \\
\hline MS 19 & 49 & M & Caucasian & $\mathrm{RR}$ & $\beta$-interf & & 1 \\
\hline MS 20 & 45 & $\mathrm{~F}$ & Caucasian & $\mathrm{RR}$ & no & & \\
\hline MS 21 & 41 & $\mathrm{~F}$ & Caucasian & & no & & 2 \\
\hline MS 22 & 42 & $M$ & Caucasian & $\mathrm{RR}$ & $\beta$-interf & & 1 \\
\hline Subject & Age & Gender & Ethnicity & Art & & Duration on art & Viral load \\
\hline \multicolumn{8}{|c|}{ (C) INFORMATION ON HIV PATIENTS } \\
\hline HIV 1 & 46 & M & Greek & no & & - & 30,010 \\
\hline HIV 2 & 31 & M & Jamaican & no & & - & 608 \\
\hline HIV 3 & 48 & M & White British & Tdf, FTC, DRV, Rit & & 4 years & $<40$ \\
\hline HIV 4 & 49 & $\mathrm{~F}$ & White British & ABC, ЗTC, EFZ & & 13 years & $<40$ \\
\hline HIV 5 & 34 & $\mathrm{~F}$ & Filipino & no & & - & 106,361 \\
\hline HIV 6 & 42 & M & White British & Tdf, FTC, EFZ & & 3 years & $<40$ \\
\hline HIV 7 & 57 & M & White British & Tdf, FTC, EFZ & & 9 years & $<40$ \\
\hline HIV 8 & 47 & $\mathrm{~F}$ & White British & AZT, ЗTC, EFZ & & 9 years & $<40$ \\
\hline HIV 9 & 44 & M & White British & Tdf, FTC, DRV, Rit & & 12 years & $<40$ \\
\hline
\end{tabular}

$R R$, relapsing-remitting; $P P$, primary-progressive; $S P$, secondary-progressive. ARTS (antiretroviral treatments):

NRTIs: ABC: Abacavir, AZT: Zidovudine, 3TC: Lamivudine, FTC: Emtricitabine.

NtRTI: Tdf: Tenofovir disoproxil.

NNRTIs: EFZ: Efavirenz.

PIs: DRV: Darunavir, Rit: Ritonavir.

Subject HC 15 (italics) was used as reference for relative quantification of MSRV/HERV-WenV, TLR4 and TLR2 gene expression. 
The mean Ct values of MSRV//HERV-Wenv, TLR4 or TLR2 were normalized compared to the Ct value of HMBS. Calculation of the relative amounts of MSRV//HERV-Wenv, TLR4 or TLR2 was performed using the $2^{\Delta \Delta C t}$ method with one $\mathrm{HC}$ used as a standard $\left(\mathrm{HC}_{15}\right.$, selected for the high amount of RNA concentration). The reference sample $\mathrm{HC}_{15}$ was analyzed in all the different plates as an inter-run calibrator. Any change in gene expression between $\mathrm{HC}, \mathrm{MS}$ and HIV patients compared to $\mathrm{HC}_{15}$ was expressed as a fold change using the formula below:

Fold increase for each patient compared to the $\mathrm{HC}_{15}=2^{\Delta \Delta \mathrm{Ct}}$

Where for each patient:

$$
\begin{aligned}
& \Delta \Delta C t=\left[(C t G I-C t H M B S)-\Delta C t H C_{15}\right] \\
& \mathrm{GI}=\mathrm{MSRV} \text { env }, \mathrm{TLR} 4, \mathrm{TLR} 2 \\
& \Delta \text { CtHC }_{15}=\Delta \text { Ct reference gene }\left(H C_{15}\right) \\
&=\left(\text { Ct } \text { GIHC }_{15}-\right.\text { CtHMBSHC} \\
&
\end{aligned}
$$

\section{Extracellular FC Staining for HERV-W}

Peripheral blood mononuclear cells (PBMC) were isolated from blood donors using Ficoll density gradient centrifugation. Surface staining for Pe-Cy7 anti-CD3 (UCHT1), PE anti-CD14 (M5E2), and Pe-Cy5 anti-CD20 (2H7) all from BD was performed on PBMC. HERV-W was detected using primary anti-HERVWEnv rabbit polyclonal antibody (Allele Biotech) with secondary CF488A goat anti-rabbit antibody (Sigma-Aldrich). A control with only secondary CF488A goat anti-rabbit antibody was included, and Fluorescence minus one (FMO) samples were used to set the gating. Cells were incubated in the dark for $30 \mathrm{~min}$ at $4^{\circ} \mathrm{C}$. After incubation, cells were washed twice with FACS buffer and centrifuged at $300 \mathrm{~g}$ for $6 \mathrm{~min}$. Cells were then fixed with 500 $\mu l$ Fixation buffer (2\% paraformaldehyde, BD). Blue Live/dead marker (ThermoFisher Scientific) was included in the staining to assess the viability. Cells were analyzed by FC using LSRII flow cytometer (BD Biosciences, USA) and FlowJo software (version V10, FlowJo, LLC, United States).

\section{Activation of B Cells With CpG and Generation of LCL}

CD20+ B cells were purified from PBMC by positive selection using a CD20+ cell isolation kit (Miltenyi Biotec) following the manufacturer's instructions. Collected CD20+ and CD20- cells were counted with haemocytometer. Purity of CD20+ cells was assessed using an anti-CD20 Ab by FC. $10^{6}$ CD20+ isolated B cells were cultured in $400 \mu \mathrm{l}$ of complete medium [Roswell Park Memorial Institute medium (RPMI) with $10 \%$ fetal calf serum (FCS), 100 units penicillin-1 $\mathrm{mg} / \mathrm{ml}$ streptomycin (pen/strep), $20 \mathrm{mM}$ L-glutamine all from Sigma- Aldrich] with $30 \mu \mathrm{g} / \mathrm{ml}$ of CpG OND 2006 (Invivogen, sequence "TCG TCG TTT TGT CGT TTT GTC GT") in 48-wells plate for $24 \mathrm{~h}$. Activation of CD20+ cells was assessed using an anti-CD86 Ab by FC.

Five to $10 \times 10^{6}$ of isolated $\mathrm{PBMC}$ were centrifuged at $300 \mathrm{~g}$ for $10 \mathrm{~min}$. Three $\mathrm{ml}$ of supernatant from a B95.8 EBV infected marmoset B cell line (19) (kindly donated by Jill Brooks, Birmingham, UK) was collected from the same batch and centrifuged at $200 \mathrm{~g}$ for $5 \mathrm{~min}$. The B95.8 supernatant was filtered through a $0.45 \mu \mathrm{m}$ filter on the PBMC pellet, and the PBMC mixed with the virus were incubated overnight at $37^{\circ} \mathrm{C}$. After centrifugation, the supernatant was discarded, and infected PBMC were re-suspended in complete RPMI $+1 \mu \mathrm{g} / \mathrm{ml}$ Cyclosporine A (Sigma). LCL was established from 6 different healthy donors and was expanded in culture.

\section{Drug Treatment}

Reverse transcriptase inhibitors inhibit HIV reverse transcription, and are divided into Nucleoside Reverse Transcriptase Inhibitors (NRTI), Nucleotide Reverse Transcriptase Inhibitors (NtRTI), and Non-Nucleoside Reverse Transcriptase Inhibitors (NNRTI). NRTI and NtRTI act as competitive substrate inhibitors, while NNRTI inhibits reverse transcriptase by binding to an allosteric site of the enzyme. Integrase inhibitors (II) inhibit the viral enzyme integrase, and protease inhibitors block the viral protease enzyme. Drugs were purchased from Sigma-Aldrich and dissolved in DMSO. One million LCL from different donors were treated with Lamivudine (NRTI), Tenofovir (NtRTI), Daranuvir (PI), Efavirenz (NNRTI) and Raltegravir (II) at concentrations of 10,1 , and $0.1 \mu \mathrm{M}$ for 5 days. At day 3, the medium was changed, and fresh drugs were added. The range of different doses was chosen based on datasheet indication and previous publications where similar tests in vitro were used to test antiretroviral efficacy against HIV $(20,21)$ or porcine endogenous retroviruses (22).

\section{Statistical Analysis}

The data were presented as medians and interquartile range, and statistical analysis was performed using GraphPad prism v7 as reported in the figure legends.

\section{RESULTS}

\section{Expression of MSRV/HERV-W in MS and HIV Patients}

Many studies have been reported in the literature showing the increased expression of MSRV/HERV-W in MS patients (4), but the expression of MSRV/HERV-W in HIV-infected patients has never been investigated until now. In this study, total RNA was extracted from PAXGENE tubes (total blood) of $22 \mathrm{MS}$ patients, $22 \mathrm{HC}$ and $9 \mathrm{HIV}$-infected patients (Table 1, average age $\mathrm{MS}=47.5, \mathrm{HC}=42.8, \mathrm{HIV}=44.2$ ). Relative quantification of MSRV/HERV-Wenv, TLR4 and TLR2 gene expression was assessed with real-time quantitative RT-PCR using the reference gene HMBS and the HC subject number 15 (HC 15, Table 1) as 1. The expression of MSRV/HERV-Wenv in the MS group was significantly higher compared with $\mathrm{HC}(p=0.019)$ and $\mathrm{HIV}(p<$ 0.001) (Figure 1A). MSRV/HERV-Wenv expression was similar in HIV patients and HC.

TLR4 expression was significantly higher in MS and HIV compared to $\mathrm{HC}$ (respectively $p=0.04$ and $p=0.001$ ) (Figure 1B), while TLR2 was increased only in MS patients (Figure 1C).

The HIV patients were then grouped into treated with antiretroviral drugs patients $(n=6)$ and untreated patients $(n=3$; Table 1C). No difference in MSRV/HERV-Wenv, TLR4 

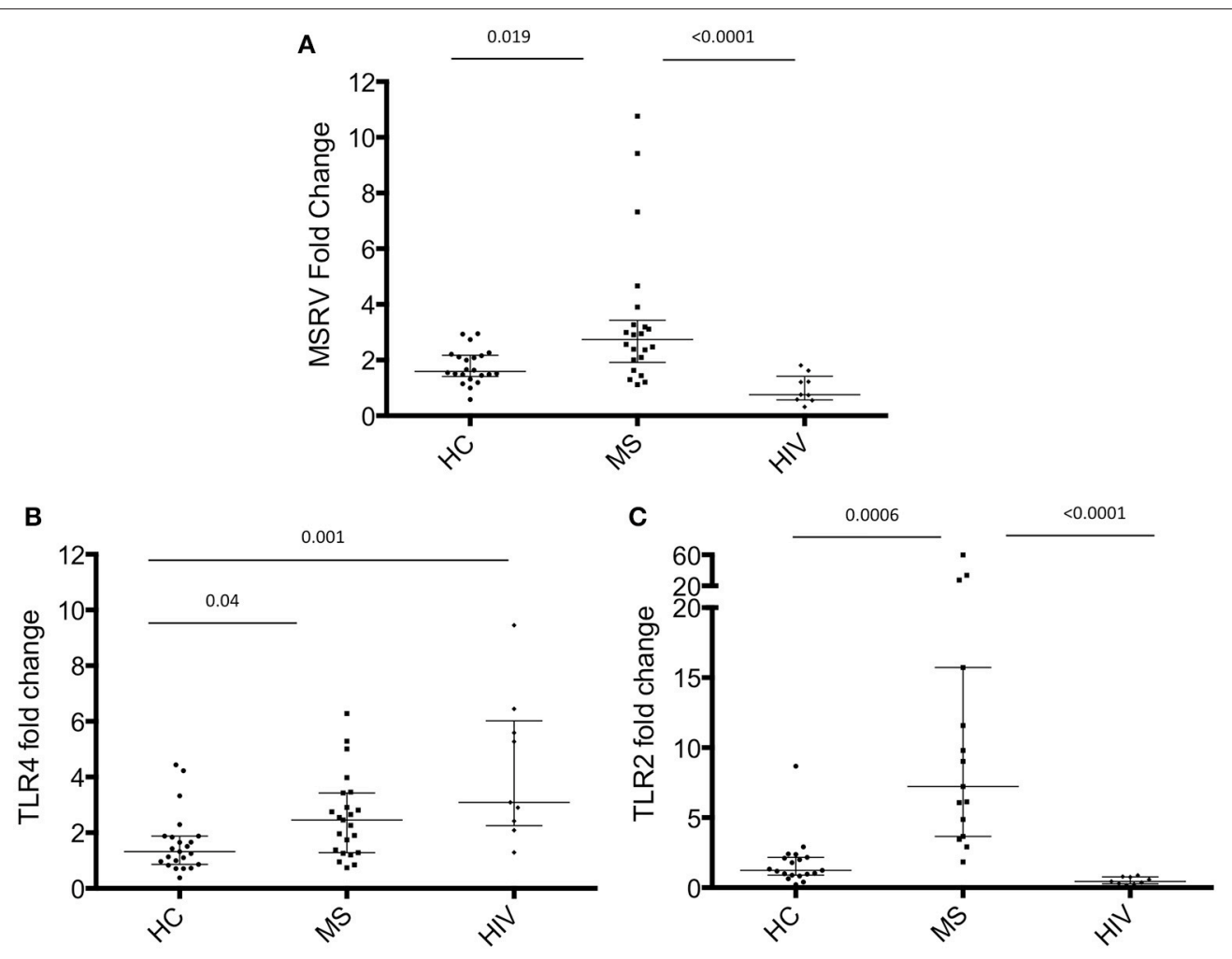

FIGURE 1 | MSRV/HERV-Wenv, TLR4 and TLR2 gene expression in HIV and MS patients and in healthy control (HC). MSRV/HERV-Wenv and TLR4 expression were evaluated by relative quantification RT-PCR using the $2^{-\Delta \Delta C t}$ method. Fold changes of (A) MSRV/HERV-Wenv, (B) TLR4, and (C) TLR2 expression of each individual sample in each group are illustrated in dots. HIV patients were compared to $\mathrm{HC}$ and MS using $\mathrm{HC} 15$ as a reference gene. Medians and interquartile range are indicated by bars (HC, $n=22$, MS, $n=22$, HIV, $n=9$; Kruskal-Wallis Test with Dunn's multiple comparison test).

and TLR2 expression between the two groups was found (Figure 2).

\section{Expression of HERV-WEnv Protein in Different Cell Types of MS and HC}

We next aimed to characterize the cells that express MSRV/HERV-W. HERV-W Env can be translated into protein and detected on the surface of cells. HERV-W Env protein expression was detected on the cell surface of immune cells from HCs using a primary anti-HERV-W Env antibody and a CF488A-labeled secondary antibody by Flow Cytometry (23). HERV-W Env was expressed by $20 \%$ of PBMC of which mainly by CD20+ B cells and CD14+ monocytes. CD3+ T cells were negative (Figure 3).

Comparison of HERV-W Env protein expression in different cell subtypes (total PBMC, T cells, B cells, and monocytes) derived from $10 \mathrm{MS}$ (6 RR-MS, 1 PP-MS, 3 SP-MS) patients and six age-matched $\mathrm{HC}$, showed no difference in HERV-W Env protein levels (Figure 3B).

EBV is strongly associated with MS (24). In vitro, the expression of MSRV/HERV-W genes/proteins is activated by some viruses such as EBV, herpes simplex virus type 1 or by influenza virus (25). One study showed that binding of the EBV surface glycoprotein gp350 could activate the expression of MSRV/HERV-W in cells from blood and brain (23). EBVimmortalized B-lymphoblastoid cell lines (LCL) were generated in vitro. LCL represents a tissue culture model for human B cell transformation and virus latency, and they express the genes of the latency III program of EBV. To investigate if EBV could activate MSRV/HERV-W, MSRV/W env RNA expression was measured in isolated CD20+ cells, CD20+ cells activated with CpG (TLR9 agonist), LCL and CD20- cells from 5 different HC donors. Quantification of MSRV/HERV-W env gene expression relative to the mean of the expression in CD20+ cells (considered as 1) was assessed with real-time RT-PCR. LCL showed 12fold increase in MSRV/HERV-W expression compared to the uninfected CD20+ cells (Figure 4A). Similarly, CpG activation induced the expression of MSRV/HERV-W env.

Relative MSRV/HERV-W env RNA expression was measured in LCL from $3 \mathrm{HC}$ and from $3 \mathrm{MS}$ to test if MS patients express more MSRV/HERV-Wenv RNA after EBV infection compared to HC. No difference was detected between LCL from HC and MS by real-time RT-PCR (Figure 4B).

\section{Expression of MSRV/HERV-W in LCL Treated With Different Antiretroviral Drugs in vitro}

MSRV/HERV-Wenv expression was then analyzed in cells treated in vitro with antiretroviral drugs. MSRV/HERV-Wenv RNA 


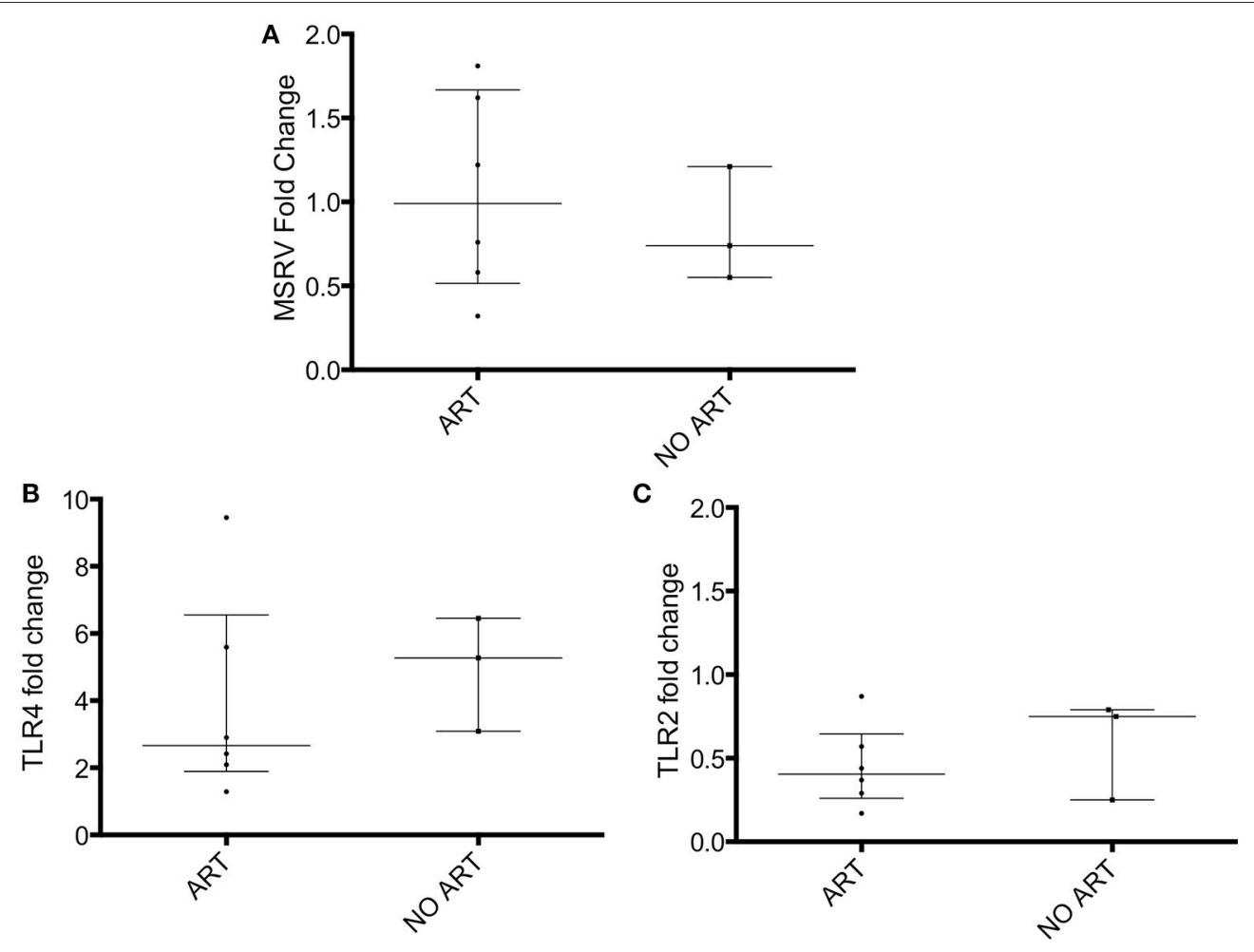

FIGURE 2 | MSRV/HERV-Wenv, TLR4 and TLR2 gene expression in HIV patients categorized in treated (ART) and untreated patients (NO ART). MSRV/HERV-WenV, TLR4 and TLR2 expression were evaluated by relative quantification RT-PCR using the $2^{-\Delta \Delta C t}$ method. Fold changes of (A) MSRV/HERV-Wenv, (B) TLR4, and (C) TLR2 expression of 9 HIV patients were categorized in treated (ART) and untreated (NO ART) patients are illustrated in dots. Medians and interquartile range are indicated by bars (ART, $n=6$, NO ART, $n=3$; Mann-Whitney Test).

expression was analyzed by RT-PCR in LCL treated with the same classes of drugs that the patients were taking. The drugs used were Lamivudine (NRTI), Tenofovir (NtRTI), Daranuvir (PI), Efavirenz (NNRTI) and Raltegravir (II). LCL from 3 HC subjects were treated with drugs at the concentrations of 10,1 , and $0.1 \mu \mathrm{M}$ for 5 days (Figure 5). Only Efavirenz (NNRTI) decreased the expression of MSRV/HERV-Wenv at the highest concentration (Figure 5D).

Cells were then treated with drug combinations, to mimic ART in vivo. Detection by RT-qPCR showed a reduced expression of MSRV/HERV-Wenv RNA (Figure 6A). Similarly, HERVW/HERV-WEnv protein showed by FC a trend of reduction in the presence of $1 \mu \mathrm{M}$ of combined drugs (Figures $6 \mathrm{~B}, \mathrm{C}$ ).

\section{DISCUSSION}

The expression of MSRV/HERV-Wenv was evaluated in a small number of MS patients, HIV-infected patients, and HC. Expression of MSRV/HERV-Wenv in MS patients was higher compared to HC, as already reported in the literature (4). HERVW Env was expressed in whole PBMC, at a high level in B cells and monocytes. No expression of HERV-W Env was detected in T cells. These results are in line with previous publications $(23,26)$ and underline a key role of B cells and monocytes in HERV-W expression. Despite increased expression of MSRV/HERV-W env
mRNA in MS patients by RT-PCR, HERV-W Env protein was detected in all samples of MS and HC using FC with no difference in the number of HERV-W positive cells between the two groups. At the protein level, no antibody has been developed yet to discriminate MSRV Env from Syn-1 (18). Moreover, the issue of HERV-W complexity extends to studies of HERV protein expression: it often remains unclear as to which genomic locus the observed HERV proteins are derived from and therefore, whether the precise identity of the protein recognized by the HERV-W Env antibody can be established. This could explain why HERV-W is expressed in similar percentage in both groups: the antibody could detect either MSRVenv or Syn-1 or both together.

As reported in the literature, EBV infection induces the expression of MSRV/HERV-W env (25). However, the activation of the retrovirus can occur not only in the presence of EBV, but also when B cells are activated with CpG. Thus, bacterial or synthetic unmethylated CpG ODNs mediate their effects by interacting with TLR9, which is expressed in $\mathrm{pDC}$ and $\mathrm{B}$ cells. Activation of $\mathrm{B}$ cells by $\mathrm{CpG}$ leads to proliferation, production of antibodies and cytokines. It is possible therefore that MSRV/HERV-W activation is induced either directly by TLR9 activation as in the presence of a bacterial infection or by pro-inflammatory cytokines expressed by CpG-activated B cells. In line with this contention, it has been reported that proinflammatory stimuli, such as IFN- $\gamma$ and TNF- $\alpha$ can activate 


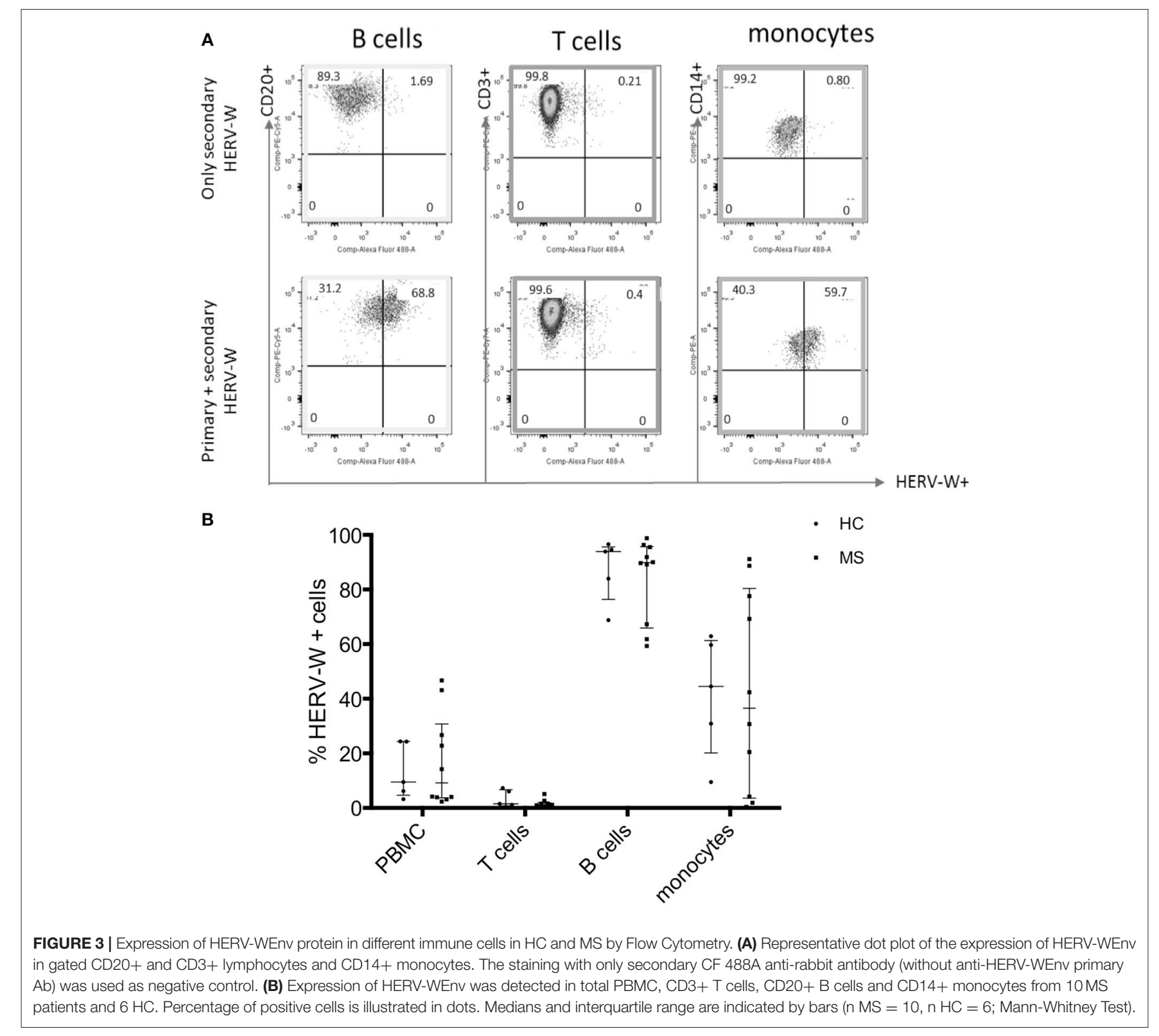

MSRV/HERV-W env expression in B cells (23). MSRV/HERV$\mathrm{W}$ env expression in LCL was not different from $\mathrm{HC}$ and people with MS, suggesting that EBV infection may potently induce expression of MSRV/HERV-Wenv and mask the original difference in MSRV/HERV-W env expression between B cells of $\mathrm{HC}$ and MS patients.

Expression of MSRV/HERV-W env in HIV-infected patients was similar to $\mathrm{HC}$ but was significantly lower than in MS patients. The relationship between exogenous and endogenous retroviruses is of relevance to human health and disease. All retroviruses have a similar genetic make-up, and homologous proteins encoded by one retrovirus could theoretically perform comparable functions for another member of the family and could thus complement a defective virus (27). Indeed, it has been reported that HIV, through its protein TAT, increases the expression of MSRV and HERV-K, as well as TLR4, in isolated $\mathrm{B}$ cells, NK cells and monocytes in vitro (28). A reduction of HERV-K expression in people who received suppressive ART has been reported $(29,30)$, but other HERV families have not been investigated. It is unclear whether HERV-K was directly inhibited by the antiretroviral drugs, or whether the loss of activation by HIV was responsible for the reduction of HERV-K viral load. It is possible that the inhibition of the RT in some way affects also the transcription of the HERV RNA. Although there is no evidence that HERV-W can function as a retrovirus capable of retrotranscription, some HERVs, and in particular HERV-K, can (31). Protease inhibitors targeted at HIV are not active against HERV$\mathrm{K}$ (32), but other classes of antiretroviral drugs, in particular the ones that target the RT enzyme, might be. Interestingly, the nonnucleoside RT inhibitors Nevirapine and Efavirenz have already 

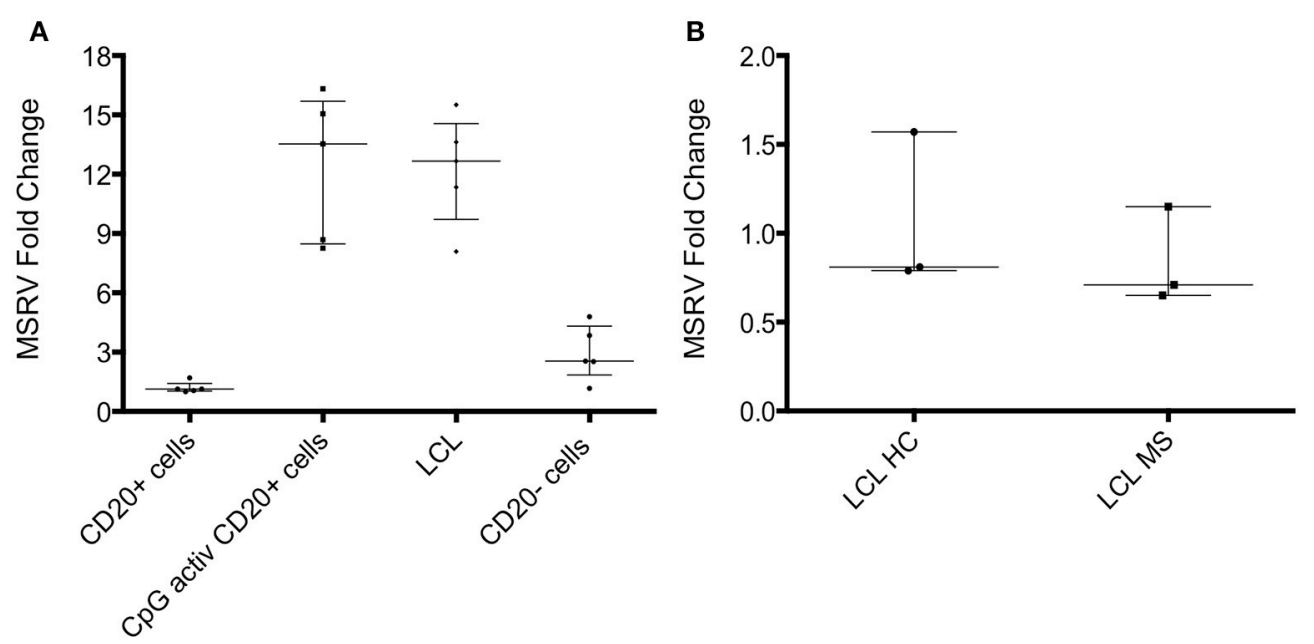

FIGURE 4 | MSRV/HERV-Wenv expression increases in LCL and CpG activated cells. (A) MSRV/HERV-Wenv expression was evaluated by relative quantification RT-PCR using the ${ }^{-}-\Delta \Delta \mathrm{Ct}$ method. Fold changes of MSRV/HERV-Wenv in isolated CD20+ cells, CpG activated CD20+ cells, LCL (B-lymphoblastoid cell line) and CD20- cells are illustrated in dots. Medians and interquartile range are indicated by bars ( $\mathrm{n}=5$, Kruskal-Wallis Test with Dunn's multiple comparison test). (B) MSRV/HERV-Wenv expression was evaluated by relative quantification RT-PCR using the $2^{-\Delta \Delta C t}$ method. Fold changes of MSRV/HERV-Wenv in LCL from HC and MS patients are illustrated in dots. Medians and interquartile range are indicated by bars ( $\mathrm{n} \mathrm{HC}=3$, n MS =3; Mann-Whitney Test).

been shown to inhibit efficiently the normal endogenous RT activity that is detectable in many human cell lines and leukemic cells $(33,34)$, suggesting a possible efficacy of this drug class in the inhibition of HERVs.

The results of this pilot study are somewhat conflicting with the original hypothesis that ART would reduce the expression of HERVs, but our data are potentially explainable with consideration of the biology of the system. HIV patients did not show an increase in HERV-W expression when compared with healthy controls. This is possibly explained by a mismatch between the cell types that produce HERV-W (primarily monocytes and B cells) and those that are affected by HIV (primarily $\mathrm{T}$ cells). In this study, RNA was extracted from HIV patients from total peripheral blood and is not representative of a specific cell type. During HIV infection, there is an alteration in immune cell populations [e.g., with B cells there is hyper activation, as well as increased apoptosis that results in B cell exhaustion (35)]. Therefore, it is possible that in HIV patients MSRV/HERV-W env expression increases in some cell subpopulations, but this change might not detectable in whole blood. Moreover, RT-qPCR may not be the best method to detect MSRV expression (4, 36, 37).

When HIV patients were divided into ART-treated and untreated, no difference was found between the two groups. However, due to the low number of HIV patients not on ART treatment $(n=3)$, we cannot finally confirm that ART in vivo does not affect MSRV/HERV-W expression.

Analyzing the patients' HIV viral load, there was no correlation between HIV viral load and MSRV/HERV-Wenv expression (Table 1). It is possible that the different ethnicities of untreated HIV patients (Jamaican, Greek, and Filipino) and those treated (all white British) could influence the expression of MSRV/HERV-W env on the basis of genetic differences. MS patients in our studies were also mainly white British.
On the other hand, TLR4 expression was higher in both MS and HIV patients compared with HC. This is potentially due to the recognition of retroviruses, endogenous for MS and exogenous for HIV, as a viral antigen by the TLR system $(7,8)$. Indeed, it has been reported that myeloid dendritic cells from HIV patients had an increased expression of TLR2 and TLR4 compared to HC (37). Hernandez et al. also showed that the expression of TLR4 in cells from untreated patients was higher than in treated and that in monocytes there was a positive correlation between both the expression of TLR2/4 and viral load, but no correlation with CD4+ T cell numbers (38). In our study TLR2 was found to be increased only in MS patients, as already reported in the literature $(39,40)$, but not in HIV patients. This might be due to the increased MSRV/HERV-W env expression (6), or to other infections (39).

In a move to identify the in vitro effects of different concentrations of drugs used by the patients on LCLs, we have recorded that only $10 \mathrm{M}$ Efavirenz (NNRTI) induced reductions in MSRV/HERV-W env expression. However, there was no clear evidence of inhibition in patients on NNRTI drugs as part of the ART mix treatment (Table 1).

When LCLs were treated with a combination of drugs a reduction of MSRV/HERV-W env RNA was detected (Figure 4A). This would suggest that a synergy between different drug classes is required to produce a measurable decrease in HERV-W expression, a contention which is in line with the demonstrable efficacy of ART against HIV as opposed to single drug therapy. Again, this effect was not seen in the HIV samples though this finding could have been accounted for by a) the mismatch between affected cells and total blood cell population described above; b) individual patient variability, and c) the small number of patients on each combination of drugs.

The number of HIV patients in this pilot study was too small to draw definite conclusions about the association of HIV 


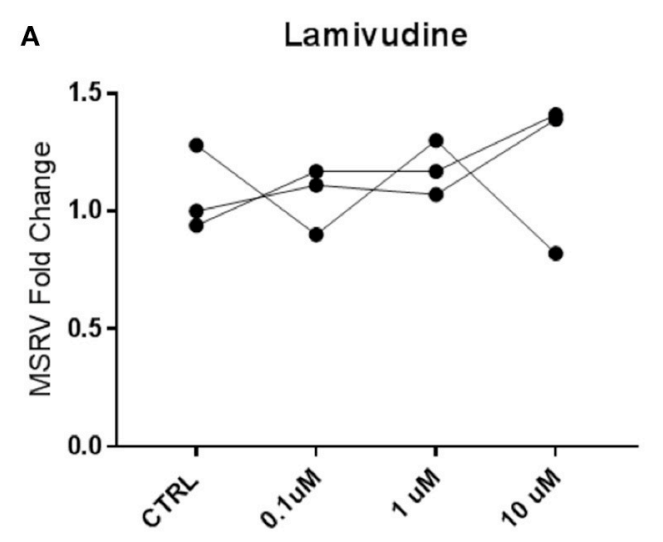

B Tenofovir
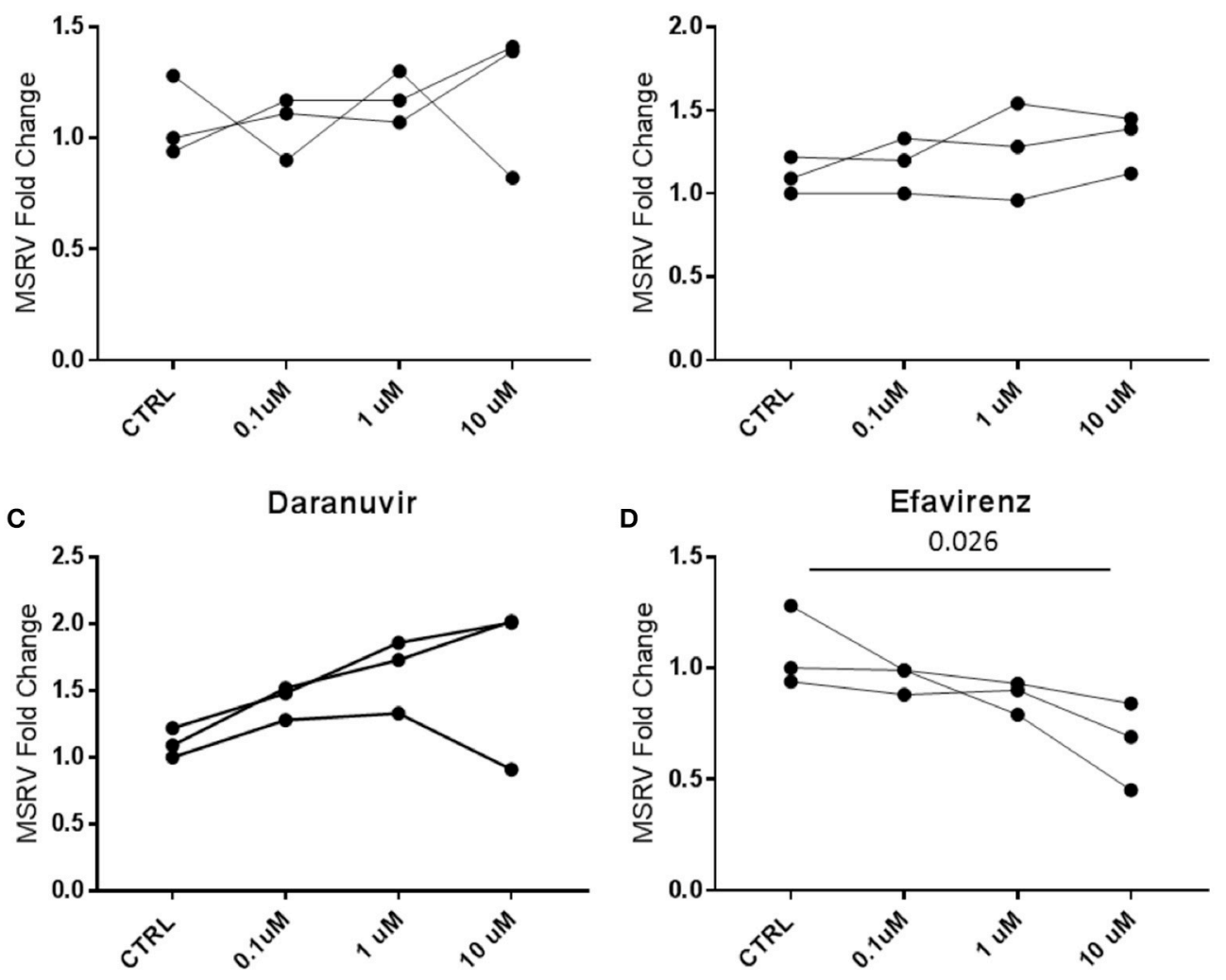

Raltegravir

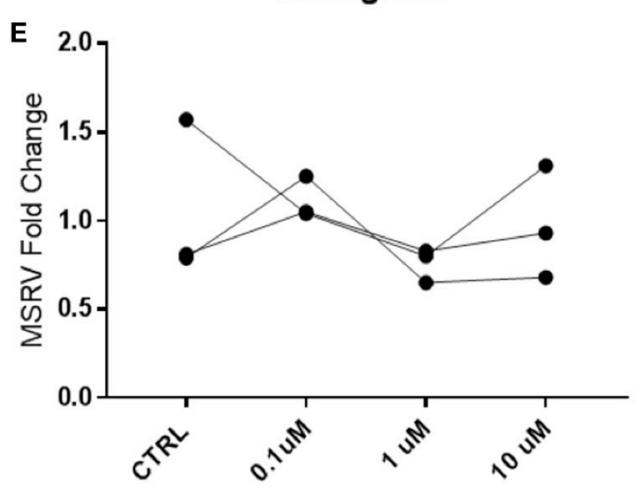

FIGURE 5 | Efavirenz decreases the expression of MSRV/HERV-Wenv. LCL from 3 different healthy donors were treated with $0.1,1$, and $10 \mu M$ of (A) Lamivudine, (B) Tenofovir, (C) Daranuvir, (D) Efavirenz, and (E) Raltegravir for 5 days. CTRL (Controls) represent LCL treated only with DMSO. MSRV/HERV-Wenv expression was evaluated by relative quantification RT-PCR using the $2^{-\Delta \Delta C t}$ method and is illustrated in dots ( $n=3$; Friedman test).

with MSRV and the role of ART. It was particularly difficult to enroll untreated HIV patients. Until larger studies are conducted, we can at this stage only speculate about these mechanisms. Therefore, larger cohorts of HIV patients are much needed to recruit to identify whether ART has a role in protecting from MS.

In conclusion, HIV-infected patients had lower expression of MSRV/HERV env than MS patients, regardless of ART status. Among ARTs, Efavirenz may reduce the expression of HERVs, warranting further investigations to clarify the potential role of ART in protecting from MS.

\section{ETHICS STATEMENT}

This study was carried out in accordance with the National Institute for Health Resaerch (NIHR) Good Clinical Practice Guidelines, with written informed consent from 
A

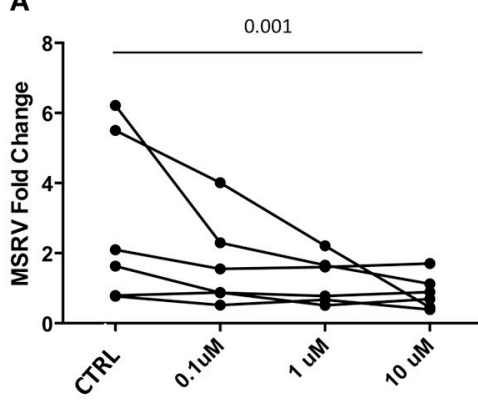

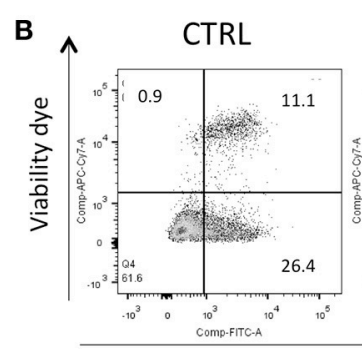

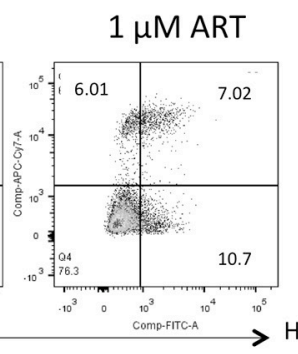

HERV-W

C

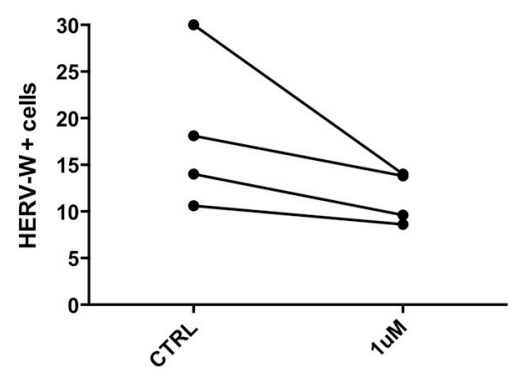

FIGURE 6 | Combined ART decreases the expression of HERV-WEnv protein. (A) LCL from 6 different donors were treated with combined Lamivudine, Tenofovir, Daranuvir, Efavirenz, and Raltegravir at 10, 1, and $0.1 \mu \mathrm{M}$ for 5 days. CTRL represents LCL treated only with DMSO. MSRV/HERV-Wenv expression was evaluated by relative quantification $R T$-PCR using the $2^{-\Delta \Delta C t}$ method. Each point represents an $L C L$ ( $n=6$; Friedman test). (B) $L C L$ were treated with combined drugs at $1 \mu M$ for 5 days. HERV-WEnv expression was evaluated by extracellular FC with dead cell marker included. One example is shown. (C) HERV-WEnv expression of 4 individual LCL is illustrated in dots before and after the treatment ( $n=4$; Wilcoxon test).

all subjects. All subjects gave written informed consent in accordance with the Declaration of Helsinki. The protocol was approved by the Nottingham Research Ethics Committee 2 (reference 08/H0408/167) and sponsored by the University of Nottingham.

\section{AUTHOR CONTRIBUTIONS}

EM conducted the experiments, analyzed the data and wrote the first draft of the paper. EM, RET, and BG designed the study. DC-T supervised the RT-PCR experiments. BG and RT provided

\section{REFERENCES}

1. Dendrou CA, Fugger L, Friese MA. Immunopathology of multiple sclerosis. Nat Rev Immunol. (2015) 15:545-58. doi: 10.1038/nri3871

2. Olsson T, Barcellos LF, Alfredsson L. Interactions between genetic, lifestyle and environmental risk factors for multiple sclerosis. Nat Rev Neurol. (2017) 13:25-36. doi: 10.1038/nrneurol.2016.187

3. Perron H, Garson JA, Bedin F, Beseme F, Paranhos-Baccala G, KomurianPradel F, et al. Molecular identification of a novel retrovirus repeatedly isolated from patients with multiple sclerosis. Proc Natl Acad Sci USA (1997) 94:7583-8.

4. Morandi E, Tanasescu R, Tarlinton RE, Constantinescu CS, Zhang W, Tench $\mathrm{C}$, et al. The association between human endogenous retroviruses and multiple sclerosis: a systematic review and meta-analysis. PLoS ONE (2017) 12:e0172415. doi: 10.1371/journal.pone.0172415

5. Gifford R, Tristem M. The evolution, distribution and diversity of endogenous retroviruses. Virus Genes (2003) 26:291-315. doi: 10.1023/A:102445 5415443 blood samples from people with RRMS and age- and gendermatched healthy controls. All the authors critically edited the manuscript and BG and RET supervised the study.

\section{ACKNOWLEDGMENTS}

We thank Dr. Pradhib Venkatesan (Department of Infectious Diseases, Nottingham University Hospitals NHS Trust) for kindly providing samples from HIV-infected patients that were either treated or not with anti-retroviral drugs. We thank Nanci Frakich for her valuable help in the laboratory.

6. Bergallo M, Loiacono E, Gambarino S, Montanari P, Galliano I, Coppo R. Tolllike receptors are essential for the control of endogenous retrovirus expression in idiopathic nephrotic syndrome minerva. Urol Nefrol. (2017) 69:201-8. doi: 10.23736/S0393-2249.16.02658-8

7. Rolland A, Jouvin-Marche E, Viret C, Faure M, Perron H, Marche PN. The envelope protein of a human endogenous retrovirus- $\mathrm{W}$ family activates innate immunity through CD14/TLR4 and promotes Th1-like responses. J Immunol. (2006) 176:7636-44.

8. Kremer D, Schichel T, Forster M, Tzekova N, Bernard C, van der Valk $\mathrm{P}$, et al. Human endogenous retrovirus type $\mathrm{W}$ envelope protein inhibits oligodendroglial precursor cell differentiation. Ann Neurol. (2013) 74:721-32. doi: 10.1002/ana.23970

9. Perron H, Dougier-Reynaud HL, Lomparski C, Popa I, Firouzi R, Bertrand JB, et al. Human endogenous retrovirus protein activates innate immunity and promotes experimental allergic encephalomyelitis in mice. PLoS ONE (2013) 8:e80128. doi: 10.1371/journal.pone.0080128

10. Gold J, Goldacre R, Maruszak H, Giovannoni G, Yeates D, Goldacre M. HIV and lower risk of multiple sclerosis: beginning to unravel a mystery using a 
record-linked database study. J Neurol Neurosurg Psychiatry (2015) 86:9-12. doi: 10.1136/jnnp-2014-307932

11. Delgado SR, Maldonado J, Rammohan KW. CNS demyelinating disorder with mixed features of neuromyelitis optica and multiple sclerosis in HIV1 infection. Case report and literature review. J Neurovirol. (2014) 20:531-7. doi: 10.1007/s13365-014-0260-8

12. Chin JH. Multiple sclerosis and HIV-1 infection: case report of a HIV controller. J Neurovirol. (2015) 21:464-7. doi: 10.1007/s13365-015-0335-1

13. Gonzalez-Duarte A, Ramirez C, Pinales R, Sierra-Madero J. Multiple sclerosis typical clinical and MRI findings in a patient with HIV infection. J Neurovirol. (2011) 17:504-8. doi: 10.1007/s13365-011-0054-1

14. Maruszak H, Brew BJ, Giovannoni G, Gold J. Could antiretroviral drugs be effective in multiple sclerosis? a case report. Eur J Neurol. (2011) 18:e110-1. doi: 10.1111/j.1468-1331.2011.03430.x

15. Chalkley J, Berger JR. Multiple sclerosis remission following antiretroviral therapy in an HIV-infected man. J Neurovirol. (2014) 20:640-3. doi: 10.1007/s13365-014-0288-9

16. Gold J, Marta M, Meier UC, Christensen T, Miller D, Altmann D, et al. A phase II baseline versus treatment study to determine the efficacy of raltegravir (Isentress) in preventing progression of relapsing remitting multiple sclerosis as determined by gadolinium-enhanced MRI: the INSPIRE study. Mult Scler Relat Disord. (2018) 24:123-8. doi: 10.1016/j.msard.2018.06.002

17. Polman CH, Reingold SC, Banwell B, Clanet M, Cohen JA, Filippi M, et al. Diagnostic criteria for multiple sclerosis: 2010 revisions to the McDonald criteria. Ann Neurol. (2011) 69:292-302. doi: 10.1002/ana.22366

18. Mameli G, Poddighe l, Astone V, Delogu G, Arru G, Sotgiu S, et al. Novel reliable real-time PCR for differential detection of MSRVenv and syncytin-1 in RNA and DNA from patients with multiple sclerosis. J Virol Methods (2009) 161:98-106. doi: 10.1016/j.jviromet.2009.05.024

19. Nagy N. Establishment of EBV-Infected lymphoblastoid cell lines. Methods Mol Biol. (2017) 1532:57-64. doi:10.1007/978-1-4939-6655-4_3

20. Volsky DJ, Li G, Hamblet N, Volsky B, Decker RS, Pellegrino MG, et al. Evaluation of multiple parameters of HIV-1 replication cycle in testing of AIDS drugs in vitro. Antiviral Res. (1992) 17:335-47.

21. Frezza C, Grelli S, Federico M, Marino-Merlo F, Mastino A, Macchi B. Testing anti-HIV activity of antiretroviral agents in vitro using flow cytometry analysis of CEM-GFP cells infected with transfection-derived HIV-1 NL4-3. J Med Virol. (2015) 88:979-86. doi: 10.1002/jmv.24418

22. Powell SK, Gates ME, Langford G, Gu ML, Lockey C, Long Z, et al. Antiretroviral agents inhibit infection of human cells by porcine endogenous retroviruses. Antimicrob Agents Chemother. (2000) 44:3432-3. doi: 10.1128/AAC.44.12.3432-3433.2000

23. Mameli G, Poddighe L, Mei A, Uleri E, Sotgiu S, Serra C, et al. Expression and activation by Epstein Barr virus of human endogenous retroviruses- $\mathrm{W}$ in blood cells and astrocytes: inference for multiple sclerosis. PLOS ONE (2012) 7:e44991. doi: 10.1371/journal.pone.0044991

24. Ascherio A, Munger KL. EBV and Autoimmunity. Curr Top Microbiol Immunol. (2015) 390:365-85. doi: 10.1007/978-3-319-22822-8_15

25. Dolei A, Perron H. The multiple sclerosis-associated retrovirus and its HERVW endogenous family: a biological interface between virology, genetics, and immunology in human physiology and disease. J Neurovirol. (2009) 15:4-13. doi: 10.1080/13550280802448451

26. Brudek T, Christensen T, Aagaard L, Petersen T, Hansen HJ, Moller-Larsen A. B cells and monocytes from patients with active multiple sclerosis exhibit increased surface expression of both HERV-H Env and HERV-W Env, accompanied by increased seroreactivity. Retrovirology (2009) 6:104. doi: 10.1186/1742-4690-6-104

27. van der Kuyl AC. HIV infection and HERV expression: a review. Retrovirology (2012) 9:6. doi: 10.1186/1742-4690-9-6

28. Uleri E, Mei A, Mameli G, Poddighe L, Serra C, Dolei A. HIV Tat acts on endogenous retroviruses of the $\mathrm{W}$ family andthis occurs via
Toll-like receptor 4: inference for neuroAIDS. AIDS (2014) 28:2659-70. doi: 10.1097/QAD.0000000000000477

29. Laderoute MP, Giulivi A, Larocque L, Bellfoy D, Hou Y, Wu HX, et al. The replicative activity of human endogenous retrovirus K102 (HERV-K102) with HIV viremia. AIDS (2007) 21:2417-24. doi: 10.1097/QAD.0b013e3282f14d64

30. Contreras-Galindo R, Gonzalez M, Almodovar-Camacho S, GonzalezRamirez S, Lorenzo E, Yamamura Y. A new Real-Time-RT-PCR for quantitation of human endogenous retroviruses type K (HERV-K) RNA load in plasma samples: increased HERV-K RNA titers in HIV-1 patients with HAART non-suppressive regimens. J Virol Methods (2006) 136:51-7. doi: 10.1016/j.jviromet.2006.03.029

31. Hohn O, Hanke K, Bannert N. HERV-K(HML-2), the best preserved family of HERVs: endogenization, expression, and implications in health and disease. Front Oncol. (2013) 3:246. doi: 10.3389/fonc.2013.00246

32. Towler EM, Gulnik SV, Bhat TN, Xie D, Gustschina E, Sumpter TR, et al. Functional characterization of the protease of human endogenous retrovirus K10: can it complement HIV-1 protease? Biochemistry (1998) 37:17137-44.

33. Sciamanna I, Landriscina M, Pittoggi C, Quirino M, Mearelli C, Beraldi R, et al. Inhibition of endogenous reverse transcriptase antagonizes human tumour growth. Oncogene (2005) 24:3923-31. doi: 10.1038/sj.onc.1208562

34. Mangiacasale R, Pittoggi C, Sciamanna I, Careddu A, Mattei E, Lorenzini $\mathrm{R}$, et al. Exposure of normal and transformed cells to nevirapine, a reverse transcriptase inhibitor, reduces cell growth and promotes differentiation. Oncogene (2003) 22:2750-61. doi: 10.1038/sj.onc.1206354

35. Moir S, Fauci AS. B cells in HIV infection and disease. Nat Rev Immunol. (2009) 9:235-45. doi: 10.1038/nri2524

36. Garson JA, Huggett JF, Bustin SA, Pfaffl MW, Benes V, Vandesompele J, et al. Unreliable real-time PCR analysis of human endogenous retrovirus-W (HERV-W) RNA expression and DNA copy number in multiple sclerosis. AIDS Res Hum Retroviruses (2009). 25:377-8. doi: 10.1089/aid.2008.0270

37. Laufer G, Mayer J, Mueller BF, Mueller-Lantzsch N, Ruprecht K. Analysis of transcribed human endogenous retrovirus $\mathrm{W}$ env loci clarifies the origin of multiple sclerosis-associated retrovirus env sequences. Retrovirology (2009) 6:37. doi: 10.1186/1742-4690-6-37

38. Hernandez JC, Stevenson M, Latz E, Urcuqui-Inchima S. HIV type 1 infection up-regulates TLR2 and TLR4 expression and function in vivo and in vitro. AIDS Res Hum Retroviruses (2012) 28:1313-28. doi: 10.1089/aid.2011.0297

39. Hossain MJ, Tanasescu R, Gran B. Innate immune regulation of autoimmunity in multiple sclerosis: focus on the role of Toll-like receptor 2. J Neuroimmunol. (2017) 304:11-20. doi: 10.1016/j.jneuroim.2016.12.004

40. Hossain MJ, Morandi E, Tanasescu R, Frakich N, Caldano M, Onion D, et al. The Soluble form of toll-like receptor 2 is elevated in serum of multiple sclerosis patients: a novel potential disease biomarker. Front Immunol. (2018) 9:457. doi: $10.3389 /$ fimmu.2018.00457

Conflict of Interest Statement: BG has received honoraria for consultancy from Merck, Roche, Biogen, and Teva UK and unrestricted research grants from Biogen Idec, Merck, Bayer Healthcare, Teva UK, Novartis, and Genzyme. None are related to this study.

The remaining authors declare that the research was conducted in the absence of any commercial or financial relationships that could be construed as a potential conflict of interest.

Copyright $\odot 2019$ Morandi, Tanasescu, Tarlinton, Constantin-Teodosiu and Gran. This is an open-access article distributed under the terms of the Creative Commons Attribution License (CC BY). The use, distribution or reproduction in other forums is permitted, provided the original author(s) and the copyright owner(s) are credited and that the original publication in this journal is cited, in accordance with accepted academic practice. No use, distribution or reproduction is permitted which does not comply with these terms. 